\title{
D-BRANE CHARGE, FLUX QUANTISATION AND RELATIVE (CO)HOMOLOGY
}

\author{
JOSÉ M FIGUEROA-O'FARRILL AND SONIA STANCIU
}

\begin{abstract}
We reconsider the problem of U(1) flux and D0-charge for D-branes in the WZW model and investigate the relationship between the different definitions that have been proposed recently. We identify the D0-charge as a particular reduction of a class in the relative cohomology of the group modulo the D-submanifold. We investigate under which conditions this class is equivalent to the first Chern class of a line bundle on the D-submanifold and we find that in general there is an obstruction given by the cohomology class of the NS 3-form. Therefore we conclude that for topologically nontrivial $B$-fields, there is strictly speaking no $\mathrm{U}(1)$ gauge field on the D-submanifold. Nevertheless the ambiguity in the flux is not detected by the D0-charge. This has a natural interpretation in terms of gerbes.
\end{abstract}

\section{INTRODUCTION}

D-branes in Lie groups have received a great deal of attention recently. They provide an ideal laboratory for the study of D-branes in nontrivial string backgrounds, as they are amenable to both microscopic analysis via the algebraic methods of boundary conformal field theory and to the more standard field theoretic techniques based on sigma models and the path integral. Moreover it is precisely in the combination of these two rather different approaches that many of the recent developments have taken place.

One of these recent developments concerns the problem of stability of D-branes and the definition and quantisation of their D0-charge [1], 2, 急, 田 5, 6, 0, 8, 9, 10], about which several somewhat different points of view seem to emerge. Our motivation in this paper is to further explore these points of view.

Throughout this paper we will refer to the submanifolds on which D-branes can wrap as D-submanifolds. In the case of the WZW model, the D-submanifolds in question are described by (twisted, shifted) conjugacy classes 11, 12, 13, 14. In general these submanifolds are not minimal (although see [15] for an example of a totally geodesic twisted conjugacy class in $\mathrm{SU}(2) \times \mathrm{SU}(2))$ and hence they are not stabilised gravitationally. This is hardly surprising as the metric is not the only

Edinburgh MS-00-010, Spin 2000/19, arXiv:hep-th/0008038. 
field in the background: there is also a $B$-field, or more precisely a closed 3 -form $H$ with integral periods.

The stability of a D-brane wrapping such submanifolds should therefore be related to this integrality condition and should manifest itself in the quantisation of the allowed D-submanifolds. There seem to be at least two mechanisms through which this may be explained. One of them [11, 16, 2, 7] uses an argument which can be understood as the vanishing of the global worldsheet anomaly [17] in the lagrangian description of the boundary WZW model. Alternatively, it was argued in [1] that the stability of these D-branes is a result of the quantisation of the flux of a U(1) gauge field on the D-brane.

As far as the definition of the D0-charge is concerned there seem to be a number of candidates. As we will recall below, the data describing a D-submanifold in a Lie group is a pair $(Q, \omega)$ where $Q$ is, in the simplest case, a conjugacy class and $\omega$ is a two-form on $Q$ such that $d \omega=H$ there. According to one view [1], 2], the D0-charge of the D-brane is defined by the flux of the gauge invariant two-form field $\omega$ on the D-submanifold. If one computes explicitly the spectrum of this D0-charge for the possible D2-branes in $\mathrm{SU}(2)$, one obtains that these charges are not quantised; however their values agree with the RR charges as obtained from the boundary state approach. This is the charge recently identified in $\|6\|$ as the brane source charge.

A second candidate definition of the D0-charge is obtained [3, 包 by adding to the brane source charge a bulk contribution, such that the resulting D0-charge agrees with the flux of a $\mathrm{U}(1)$ gauge field defined on the D-submanifold. According to this view, the quantisation of the D0-charge is a consequence of the quantisation of the flux of this $\mathrm{U}(1)$ field. In the nomenclature of [6], this charge can be identified either with the Page charge [5] or with the Maxwell charge [3], which in this case coincide. This definition of the D0-charge requires a trivialisation of the NS 3-form.

An alternative definition of the D0-charge of such a D-brane was proposed in [7] by one of the authors, where the quantisation of the D0-charge is seen as a consequence of the vanishing of the global worldsheet anomaly for the boundary WZW model. This third definition is essentially a Page charge, and one of the aims of this paper is to compare this charge with the one in [3, 5].

Let $Q$ be a D-submanifold in a compact simple Lie group $G$ and let $k$ be the level of the WZW model. Let $H$ be the bi-invariant 3form in $G$ and let $\omega$ be a 2 -form on $Q$ such that $H=d \omega$ there. We will show that the charge in [0] can be identified with the reduction modulo $k$ of the class of $(H, \omega) / 2 \pi$ in the degree 3 integral relative cohomology of $G$ modulo $Q$. On the other hand, a $U(1)$ gauge field on the D-submanifold would give rise to a class in the degree 2 integral cohomology of $Q$, namely the first Chern class of the corresponding 
line bundle. These two cohomology groups are related by a long exact sequence in cohomology and studying this sequence will reveal that the integral cohomology class represented by $H / 2 \pi$ is the obstruction to associate a line bundle on $Q$ with the relative class $[(H, \omega)] / 2 \pi$. In other words, when $H$ is topologically nontrivial there is strictly speaking no line bundle on the D-submanifold; but rather, as we will see, an equivalence class of line bundles corresponding to the reduction modulo $k$ of their first Chern classes, in such a way that their fluxes also compute the D0-charge. This has a natural interpretation in terms of gerbes.

This paper is organised as follows. In Section 2 we briefly review the geometry of the WZW model with and without boundary, paying particular attention to the consistency conditions which must be met for the well-definedness of the quantum theory. The appropriate mathematical framework for discussing these conditions is the relative cohomology of the Lie group modulo the D-submanifold. In Section 3 we study the relation between the D0-charge defined in [7] and flux quantisation. We will find that despite the absence in general of a line bundle on the D-submanifold, the modularity of the D0-charge is such that the flux also computes it. This modularity has a natural interpretation in terms of gerbes. Finally in Section 1 we summarise the main points of the paper.

\section{ACKNOWLEDGEMENTS}

It is a pleasure to thank Costas Bachas, Michael Douglas, Krzysztof Gawędzki, Maximilian Kreuzer, Donald Marolf, Andrew Ranicki, Andreas Recknagel, Elmer Rees, Christoph Schweigert, Michael Singer and Arkady Tseytlin for correspondence. In addition JMF would like to thank the Spinoza Institute and particularly Bernard de Wit for the kind hospitality while this paper was being written. This work was finished while on a visit to CERN.

\section{D-BRANES IN THE BOUNDARY WZW MODEL}

In this section we describe the boundary WZW model; that is, the WZW model associated to a worldsheet with boundary [18, 16]. It is instructive to compare this with the "standard" WZW model, so we recall this briefly first. Although these results are not new, we believe it is useful to collect them here in a hopefully simpler form than has been done until now.

2.1. The WZW model. Let $\Sigma$ be a compact Riemann surface (without boundary) and let $G$ be a Lie group admitting a bi-invariant metric. In order to circumvent special cases associated with abelian groups or with noncompact groups, we will assume that $G$ is compact semisimple; although the theory is of course more general. The WZW model 
is the theory of maps $g: \Sigma \rightarrow G$ defined by the following action:

$$
I=\int_{\Sigma}\left\langle g^{-1} \partial g, g^{-1} \bar{\partial} g\right\rangle+\int_{M} H
$$

where $M$ is a 3-dimensional submanifold of $G$ with boundary $\partial M=$ $g(\Sigma)$, and $H=\frac{1}{6}\langle\theta,[\theta, \theta]\rangle$, where $\theta$ is the left-invariant Maurer-Cartan 1-form on $G$ and $\langle-,-\rangle$ is an invariant metric on the Lie algebra of $G$.

There is an obstruction to the existence of $M$, which is measured by the homology class of $g(\Sigma)$ in $H_{2}(G)$. Demanding that this class vanish for all $g(\Sigma)$ is equivalent to demanding that $H_{2}(G)$ vanishes. This is the case, for instance, for $G$ a compact semisimple Lie group.円

Even when the obstruction is overcome and such an $M$ found, the action depends on the choice of $M$; although because the 3-form $H$ is closed, the equations of motion do not. Indeed if $M^{\prime}$ is another 3dimensional submanifold of $G$ with boundary $g(\Sigma)$, then $M-M^{\prime}$ is a 3-cycle and as observed originally in [19], the path integral is also independent on the choice of extension provided that

$$
\int_{M-M^{\prime}} H \in 2 \pi \mathbb{Z} .
$$

Demanding that this be the case for all 3-cycles $M-M^{\prime}$, is equivalent to the cohomology class $[H] / 2 \pi \in H^{3}(G ; \mathbb{R})$ being integral. The definition of $H$ (as well as the kinetic term) involves a choice of bi-invariant metric. For $G$ a simple Lie group there is a unique conformal class of bi-invariant metrics, and it is always possible to choose a metric in this class for which this integrality condition is satisfied.

2.2. The boundary WZW model. Suppose now that $\Sigma$ is a compact Riemann surface with nonempty boundary $\partial \Sigma$. The boundary is homeomorphic to a disjoint union of circles. We will assume for simplicity of exposition that the boundary is connected, so that it consists of only one circle. The extension to the general case does not represent any added difficulties nor does it reveal any extra structure at this level.

Dirichlet boundary conditions in the WZW model are described 18 by a submanifold $\iota: Q \hookrightarrow G$ of the Lie group and a 2 -form $\omega$ on $Q$ such that $\iota^{*} H=d \omega$. The submanifold $Q$ is called a D-submanifold. The WZW model corresponding to this boundary condition is the theory of maps $g: \Sigma \rightarrow G$ sending the boundary $\partial \Sigma$ to $Q$, which is governed by

\footnotetext{
${ }^{1}$ For compact Lie groups where $H=0$ or noncompact groups where $H$ is exact, one can work with the $B$-field directly, without having to introduce the submanifold $M$ and hence avoiding any obstructions. For these theories, it is the $B$-field that is part of the data and one does not impose that the theory be independent of this choice.
} 
the following action

$$
I=\int_{\Sigma}\left\langle g^{-1} \partial g, g^{-1} \bar{\partial} g\right\rangle+\int_{M} H-\int_{D} \omega
$$

where $M$ is a 3-dimensional submanifold of $G$ with boundary $\partial M=$ $g(\Sigma)+D$ where $D$ is a 2-dimensional submanifold of $Q$. Applying the boundary again, we see that $g(\Sigma)$ and $D$ have the same boundary with opposite orientations. Therefore we can think of $\partial M$ as the manifold $g(\Sigma) \cap_{\partial} D$ obtained by gluing the worldsheet $g(\Sigma)$ and $D$ along their common boundary, as in Figure 11.

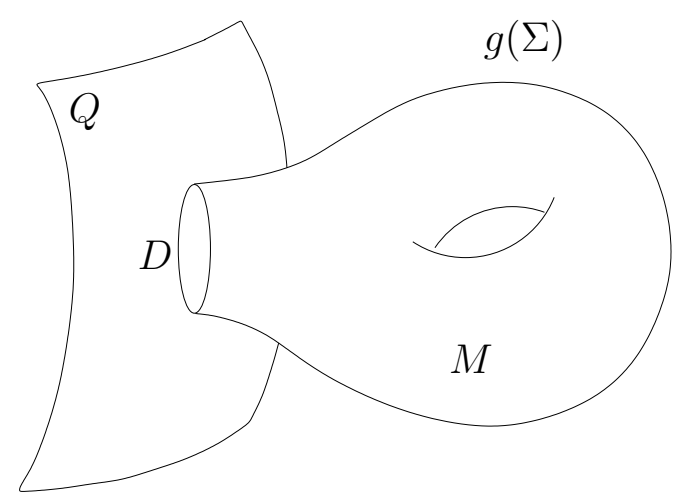

FiguRE 1. The relation $\partial M=g(\Sigma)+D$. In the figure $M$ is the solid object whose boundary is $g(\Sigma)+D$, where $D$ is contained in the D-submanifold $Q$.

As in the case of the WZW model, there is a homological obstruction to the existence of $M$. This time the relevant homology theory is the relative homology of $G$ modulo $Q$. Since the boundary of the worldsheet $g(\Sigma)$ of the string lies in $Q, g(\Sigma)$ is a relative 2-cycle and defines a homology class in $H_{2}(G, Q)$. The existence of $M$ and $D \subset Q$ such that $\partial M=g(\Sigma)+D$ simply says that $g(\Sigma)$ ia a boundary modulo $Q$, whence its relative homology class is zero. Demanding that this be true for all $g(\Sigma)$ is equivalent to demanding that $H_{2}(G, Q)$ vanish.

Even when the existence of $M$ (and hence $D$ ) is unobstructed, such $M$ is generally not unique and the action will depend on the choice of $M$. As in the WZW model, the equations of motion do not depend on the choice of $M$. (In fact, they do not depend on $\omega$ either, only the boundary conditions do.) A condition which guarantees the independence of the path integral on the choice of $M$ can again be captured cohomologically - this time in the relative cohomology of the pair $(G, Q)$ 18, 16.

To see this let $M_{1}$ and $M_{2}$ be two 3-dimensional submanifolds of $G$ such that $\partial M_{1}=g(\Sigma)+D_{1}$ and $\partial M_{2}=g(\Sigma)+D_{2}$ for some $D_{1}$ and 

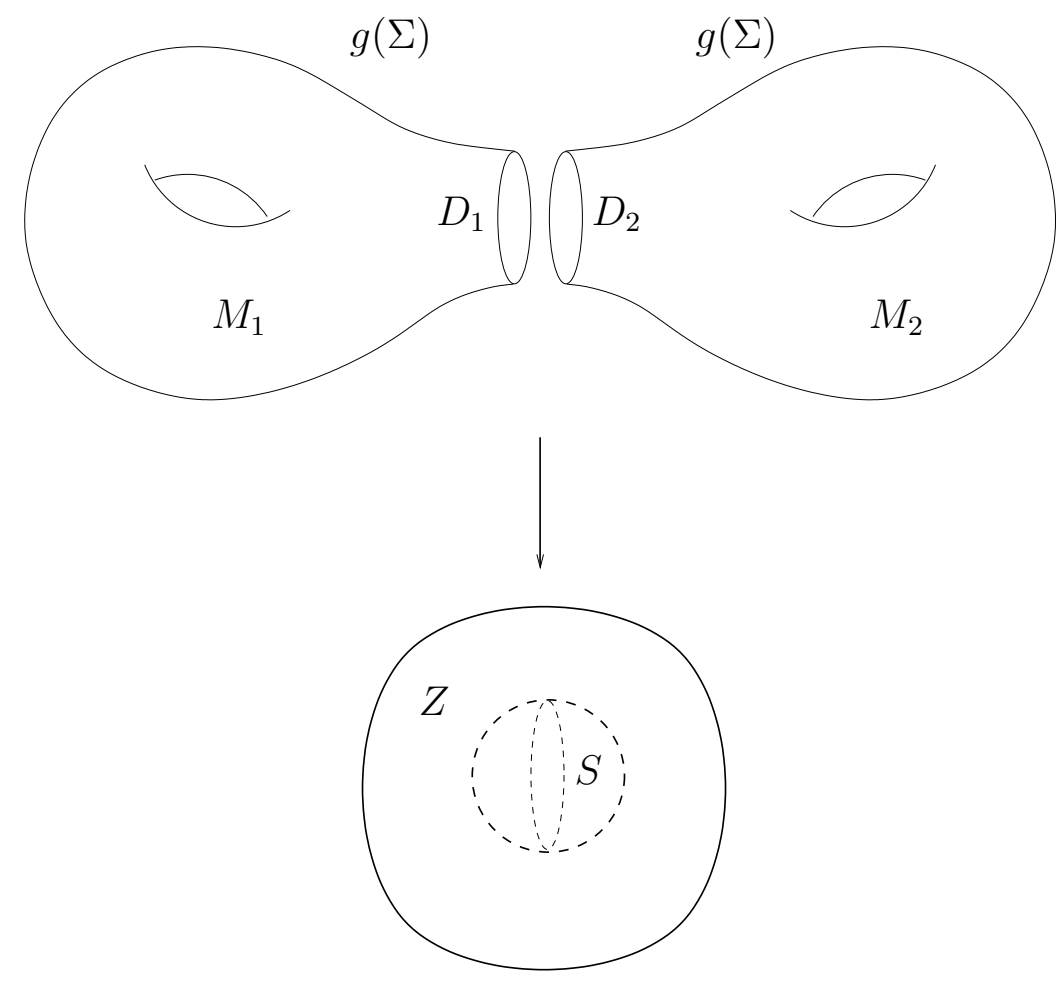

Figure 2. Gluing $M_{1}$ and $M_{2}$ along $g(\Sigma)$ to obtain the relative cycle $Z$, and gluing $D_{1}$ and $D_{2}$ along $g(\partial \Sigma)$ to obtain its boundary $\partial Z=S$.

$D_{2}$ in $Q$. Then the difference in the Wess-Zumino term is

$$
\left(\int_{M_{1}} H-\int_{D_{1}} \omega\right)-\left(\int_{M_{2}} H-\int_{D_{2}} \omega\right)=\int_{M_{1}-M_{2}} H-\int_{D_{1}-D_{2}} \omega .
$$

Let $Z=M_{1}-M_{2}$ and $S=D_{1}-D_{2}$. Notice that

$$
\partial Z=\partial M_{1}-\partial M_{2}=\Sigma+D_{1}-\left(\Sigma+D_{2}\right)=D_{1}-D_{2}=S .
$$

Since $S \subset Q, Z$ is a cycle modulo $Q$, whence it defines a class in the degree 3 relative homology of $G$ modulo $Q$. We can picture $Z$ as obtained by gluing $M_{1}$ and $M_{2}$ along $g(\Sigma)$ and $S$ as gluing $D_{1}$ and $D_{2}$ along their boundary $g(\partial \Sigma)$, as shown in Figure 2 .

The path integral is independent of the choice of $Z$ (and hence $S$ ) provided that the quantity

$$
C=\frac{1}{2 \pi}\left(\int_{Z} H-\int_{S} \omega\right)
$$

is integral. As we now explain, this is simply the pairing between the relative homology and the relative cohomology of $G$ modulo $Q$ (see, e.g., 20, 21]. 
The real relative cohomology of $G$ modulo $Q$ can be computed from the relative de Rham complex. A relative $p$-form in this complex consists of a pair $(\alpha, \beta)$ where $\alpha$ is a $p$-form on $G$ and $\beta$ is a $(p-1)$-form on $Q$. The differential is defined by $d(\alpha, \beta)=\left(d \alpha, \iota^{*} \alpha-d \beta\right)$ where $\iota: Q \rightarrow G$ is the embedding and $\iota^{*}$ is the pullback on forms. It obeys $d^{2}=0$ and the resulting cohomology is the relative de Rham cohomology $H^{*}(G, Q ; \mathbb{R})$ of $G$ modulo $Q$. Notice that a relative form $(\alpha, \beta)$ is closed if $\alpha$ is closed in $G$ and exact when restricted to $Q: \iota^{*} \alpha=d \beta$.

As in the de Rham complex, integration provides the pairing between cycles (more generally, currents) and closed forms. Given a relative $p$ cycle $N$ and a closed relative $p$-form $(\alpha, \beta)$ the expression

$$
\int_{N} \alpha-\int_{\partial N} \beta
$$

gives a pairing between the relative homology and the relative de Rham cohomology of $G$ modulo $Q$.

Therefore we see that the quantity $C$ in equation (3) is precisely the result of the pairing between the relative 3 -cycle $Z$ and the relative 3 -form $(H, \omega) / 2 \pi$. Demanding that $C$ be integral for all relative cycles $Z$ is simply the requirement that the relative cohomology class $[(H, \omega)] / 2 \pi \in H^{3}(G, Q ; \mathbb{R})$ be integral, which is precisely the cancellation of the global worldsheet anomaly [17]. Table 11 contrasts the topological conditions for the existence of the quantum WZW models with and without boundary.[?

\begin{tabular}{|c|c|c|}
\hline Model & Obstruction & Well-definedness \\
\hline \hline WZW & $H_{2}(G)=0$ & {$[H] / 2 \pi \in H^{3}(G ; \mathbb{Z})$} \\
BWZW & $H_{2}(G, Q)=0$ & {$[(H, \omega)] / 2 \pi \in H^{3}(G, Q ; \mathbb{Z})$} \\
\hline
\end{tabular}

TABLE 1. Topological conditions for the existence of the quantum WZW model with and without boundary.

In a theory of strings propagating in a Lie group, we want both closed strings propagating in the bulk and open strings with ends in the Dbranes. Therefore consistency of the theory requires that both the WZW model and the boundary WZW model should be well-defined. This means that both $H_{2}(G)$ and $H_{2}(G, Q)$ should vanish; and that both $[H] / 2 \pi \in H^{3}(G ; \mathbb{R})$ and $[(H, \omega)] / 2 \pi \in H^{3}(G, Q ; \mathbb{R})$ should be integral classes.

\footnotetext{
${ }^{2}$ This table possibly suggests that the boundary WZW model should be renamed the relative WZW model. Alas we have not been able to reach an agreement on this point.
} 
2.3. D-branes in WZW models. An interesting class of D-branes which are by now well-understood are those where the D-submanifold $Q$ corresponds to a (possibly twisted, shifted) conjugacy class. These D-branes are special in that they preserve not just conformal invariance but also (one half of) the infinite-dimensional symmetry current algebra of the WZW model. They are described in terms of the following gluing conditions:

$$
J=R \bar{J},
$$

where $J=-\partial g g^{-1}, \bar{J}=g^{-1} \bar{\partial} g$ and $R$ is a metric-preserving automorphism of the Lie algebra of $G$. This type of gluing conditions describe [11, 12, 13, 14] D-branes whose worldvolumes lie on twisted conjugacy classes

$$
C_{r}\left(g_{0}\right):=\left\{r(g) g_{0} g^{-1} \mid g \in G\right\},
$$

where $r: G \rightarrow G$ is the metric-preserving automorphism of $G$ which integrates $R$.

Two metric-preserving automorphisms $r$ and $r^{\prime}$ which are related by an inner automorphism, yield twisted conjugacy classes $C_{r}\left(g_{0}\right)$ and $C_{r^{\prime}}\left(g_{0}\right)$ which are simply shifted relative to each other. Hence in this sense these types of D-submanifolds are classified |22 by the group Out $_{o}(G)$ of metric-preserving outer automorphisms of $G$, which is defined as the quotient $\operatorname{Aut}_{o}(G) / \operatorname{Inn}_{o}(G)$ of the group of metric-preserving automorphisms by the invariant subgroup of inner automorphisms.

As shown in [11, 16, 7] there is a natural 2 -form $\omega$ on each twisted conjugacy class $C_{r}\left(g_{0}\right)$ such that $d \omega$ agrees with the restriction of the 3 -form $H$ to $C_{r}\left(g_{0}\right)$. This 2 -form is obtained by demanding that the boundary conditions associated to the gluing conditions (1) and the ones coming from the sigma model description of the WZW model coincide.

\section{Flux QuANTiSATion AND D0-CHARGE}

The issue of flux quantisation and the definition of the D0-charge for D-branes in Lie groups has generated a great deal of interest recently. The existence of a U(1) gauge field on the D-brane whose flux is quantised was assumed in [1], 3月, in order to discuss the stability and D0-charge of the D2-branes in SU(2). Alternatively, it was shown in [0] that both the stability and the various D0-charges of this type of Dbranes can be analysed without having to rely on the existence of a $\mathrm{U}(1)$ gauge field on the D-brane, by defining all the relevant quantities in terms of the globally defined gauge invariant fields $H$ and $\omega$. The central concept in this approach is the global worldsheet anomaly, whose vanishing explains both the discrete spectrum of stable D-branes and the quantisation of a suitably defined D0-charge. However one question 
remains: Does the vanishing of the worldsheet anomaly actually imply the quantisation of a $U(1)$ gauge field flux on the brane?

This question is symbolically encoded in the following identity:

$$
\int_{Z} H-\int_{S} \omega \stackrel{?}{=} \int_{S} F ;
$$

where the precise definition and geometrical nature of the field $F$ in the right-hand side have hitherto remained somewhat obscure. The purpose of this section is to shed some light on this hypothetical equivalence.

Before going into any systematic analysis let us make a rather simple but instructive remark. The left-hand side of equation (可) depends not only on $S$ but also on $Z$, whereas the right-hand side depends on $S$ and on $F$. Given an $S$ and a $Z$, it is possible to find an $F$ such that the identity in (5) holds; but this $F$ will depend on $Z$. The existence of one $F$ for which this identity holds irrespective of $Z$, means that the $Z$-dependence of the left-hand side is only apparent and this imposes a condition on $H$. To see this let $Z^{\prime}$ be another submanifold with boundary $S$. The identity in (5) would result in the following condition on $H$

$$
\int_{Z-Z^{\prime}} H=0
$$

where $Z-Z^{\prime}$ is a 3 -cycle, and this would imply that $H$ is exact. This suggests that when $H$ is not exact, the identity in (5) will not hold for a fixed $F$.

3.1. A line bundle on the D-submanifold. Clearly, the strong version of the statement encoded in (5) would be to have a line bundle on the D-submanifold whose Chern class is equal to the relative class defined by the fields $H$ and $\omega$. As we now explain, there are conditions under which the relative class represented by $(H, \omega) / 2 \pi$ is equivalent to an integral class in $H^{2}(Q)$; that is, to the Chern class of a line bundle on the D-submanifold $Q$. In this case, $C$ in (3) can be understood as the flux of $F / 2 \pi$, where $F$ is the curvature on the line bundle.

Indeed, suppose that $H=d B$ is exact. This does not mean that the relative cocycle $(H, \omega)$ is a coboundary. Indeed, the cocycle condition simply says that $\iota^{*} H=\iota^{*} d B=d \omega$ on $Q$, whence the 2 -form $F=$ $\iota^{*} B-\omega$ defined on $Q$ is closed and defines a class in $H^{2}(Q)$. If $W$ is any 3-dimensional submanifold of $G$ whose boundary $\partial W$ is contained in $Q$, then using Stokes we have that

$$
\int_{W} d B-\int_{\partial W} \omega=\int_{\partial W} F
$$


and hence integrality of $[(d B, \omega)] / 2 \pi \in H^{3}(G, Q ; \mathbb{R})$ is precisely the integrality of $[F] / 2 \pi \in H^{2}(Q ; \mathbb{R})$. [ This is equivalent to the existence of a line bundle on $Q$ whose curvature is $F$ and whose first Chern class is $[F] / 2 \pi$.

Furthermore, the converse is also true and a relative class $[(H, \omega)]$ in $H^{3}(G, Q)$ comes from a class in $H^{2}(Q)$ only if the integral class represented by $H / 2 \pi$ is zero. th This is a consequence of the exact cohomology sequence (see, e.g., [21])

$$
\cdots \rightarrow H^{2}(G) \stackrel{\iota^{*}}{\rightarrow} H^{2}(Q) \rightarrow H^{3}(G, Q) \rightarrow H^{3}(G) \rightarrow \cdots
$$

Indeed, consider a relative class $[(H, \omega)] / 2 \pi$ in $H^{3}(G, Q)$. By exactness at $H^{3}(G, Q)$, such a class comes from a class in $H^{2}(Q)$ - that is, is in the image of $H^{2}(Q) \rightarrow H^{3}(G, Q)$-if and only if it is the kernel of $H^{3}(G, Q) \rightarrow H^{3}(G)$; in other words if the class $[H] / 2 \pi$ in $H^{3}(G)$ vanishes.

In other words, we can understand the integral cohomology class in $H^{3}(G)$ represented by $H / 2 \pi$ as the obstruction to defining a line bundle $L$ on $Q$ whose first Chern class obeys $c_{1}(L)=[(H, \omega)] / 2 \pi$. From this it follows in particular that, for a compact semisimple Lie group, there is no line bundle on a D-brane described by a conjugacy class.

3.2. Local considerations. It is important to remark that even if $H$ is not exact, its restriction to $M$ is. This follows from the fact that a top form on a manifold with nonempty boundary is exact, and the fact that $H$ is a 3 -form and $M$ is a 3 -manifold with nonempty boundary $g(\Sigma) \cup_{\partial} D$. Therefore there exists a 2-form $B$ on $M$ such that $H=d B$. This means that there is a closed 2-form $F=B-\omega$ on $D$ such that the equation (5) is satisfied. The triviality of $H^{3}(M)$ is true also in integral cohomology, therefore there is a line bundle on $D$ whose curvature is $F$, and the integrality of $C$ is the quantisation of the flux of $F$. It is important to emphasise that this line bundle is defined on $D$ and not on all of $Q$. In other words, whereas $\omega$ is intrinsic to $Q, B$ is intrinsic to $M$ and hence $F$ is only defined where both of these quantities make sense; that is, on $D=M \cap Q$. This seems to suggest a physical picture of the $\mathrm{U}(1)$ field on the brane being due to the ends of the open strings ending on the D-brane and hence existing locally near them.

It might be illuminating to compare this with the case of the standard WZW model. Although $H$ is in general not an exact form on the group $G$, it again becomes exact when restricted to $M$, the 3-dimensional submanifold of $G$ whose boundary is $\partial M=g(\Sigma)$. This means that

\footnotetext{
${ }^{3}$ Strictly speaking, the above equality only proves that $[F] / 2 \pi$ is an integer on those 2-cycles in $Q$ which bound in $G$. Since we are assuming, for consistency of the WZW model that $H_{2}(G)=0$ this is true for all 2-cycles on $Q$.

${ }^{4}$ Notice that this is stronger than the fact that $H$ should be exact, since this only means that the class $[H] / 2 \pi$ is torsion. Of course, if $G$ is simply connected, $G$ is homotopy equivalent to a product of odd spheres and hence $H^{3}(G)$ has no torsion.
} 
there is a 2 -form $B$ in $M$ which satisfies $H=d B$ there. In terms of this form, the action can be written in a manifestly local way [19], since

$$
\int_{M} H=\int_{M} d B=\int_{g(\Sigma)} B=\int_{\Sigma} g^{*} B .
$$

Notice that $B$ is not generally the restriction to $M$ of a 2 -form defined on the whole group, as this would require $H$ to be exact.

3.3. D0-charge. Several definitions of the D0-charge have been proposed recently. The picture that begins to emerge is that one can in fact introduce several D0-charges [6], distinguished not only by their form but also by their specific properties (e.g., gauge invariance, quantisation). In the sigma model framework one can define at least two types of charges: the so-called brane source charge introduced in [1], which is gauge invariant but not quantised, and a Page charge [5, 7] which is gauge invariant (at least infinitesimally) and quantised.?

The brane source charge can be written as the integral of the twoform $\omega$ on a 2-cycle of the D-submanifold. It is in general not conserved because $\omega$ is not closed. Nevertheless its nonconservation is to a large extent under control since $d \omega=H$. In fact this very relation suggests us a way of modifying the brane source charge in order to obtain a conserved quantity, which turns out to be nothing but $C$. This procedure is reminiscent of the way one constructs the Page charge in supergravity. Notice however that, as we pointed out before, $C$ depends not only on $H, \omega$ and $S \subset Q$, which describe the given D-brane configuration, but it depends also on $Z$, the 3 -submanifold of $G$ with boundary $S$.

An alternative way of motivating the definition of the (Page) D0charge in terms of the quantity $C$ in (3) is the following. We have seen that $C$ agrees, when $H$ is exact, with the normalised flux of a $\mathrm{U}(1)$ gauge field $F$ on $Q$. It is therefore natural in this case to identify $C$ with the D0-charge of a region $S \subset Q: \int_{S} F / 2 \pi$. This point of view led in [7] to the proposal that the expression for $C$ in equation (3) should be understood as a covariantisation of $\int_{S} F / 2 \pi$ in the case where $H$ is not exact. However, as noted above, this expression depends on $Z$, the 3-submanifold of $G$ with boundary $S$.

Physically the D0-charge of the region $S$ should not depend on $Z$. However, as we saw above, the difference in the charges computed by $Z$ and $Z^{\prime}$ is the integral of $H / 2 \pi$ on the 3 -cycle $Z-Z^{\prime}$ of $G$. For $G$ a compact simple Lie group, $H^{3}(G) \cong \mathbb{Z}$. Therefore if the 3-cycle $Z-Z^{\prime}$ is mapped to $n$ under this isomorphism, one has

$$
\frac{1}{2 \pi} \int_{Z-Z^{\prime}} H=n k
$$

\footnotetext{
${ }^{5}$ There is a third type of charge, the Maxwell charge, which is computed in [3] and which agrees with the Page charge in the case of D2-branes in the WZW model.
} 
which is always a multiple of the level $k$. Therefore, if we insist in the $Z$-independence of the D0-charge, we must define it to be given by $C$ (with an appropriate normalisation) and reduce it modulo $k$. In other words, the D0-charge is the image of $[(H, \omega)] / 2 \pi$ under the natural map $H^{3}(G, Q ; \mathbb{Z}) \rightarrow H^{3}\left(G, Q ; \mathbb{Z}_{k}\right)$ induced by reduction modulo $k$.

In [7], for the special case of $G=\mathrm{SU}(2)$ this modularity was found to be consistent with the fact that the D0-branes sitting at the points in the center of the group should carry the same charge. This modularity of the D0-charge was also observed in [8] in the context of the supersymmetric WZW model, where the level is shifted to $k+2$ for $G=\mathrm{SU}(2)$.

For a compact semisimple Lie group $G=\prod_{i=1}^{n} G_{i}$, with $G_{i}$ simple and with level $k_{i}$, the same argument implies that the D0-charge should be defined modulo the greatest common divisor $k=\operatorname{gcd}\left(k_{1}, \ldots, k_{n}\right)$ of the levels. Notice that this has the property that if two of the levels are coprime, then the charge is always zero.

Defining the charge in this way also has the virtue that the hypothetical equality in (5) becomes an honest equality modulo $k$. In this way, one could still obtain the D0-charge from the flux of a (locally defined) $\mathrm{U}(1)$ gauge field, since the change in the flux on overlaps is a multiple of $k$ and hence not seen by the charge.

3.4. Gerbe interpretation. The fact that there is no canonical line bundle on the D-submanifold should not come as a surprise. At the heart of the WZW model is an integral class in $H^{3}(G)$, which should alert us to the existence of an underlying gerbe (see, for example, [23]). Gerbes are the third member in an infinite sequence of objects, whose first two members are smooth circle-valued functions and line bundles, respectively. They are an attempt to geometrise integral classes in $H^{3}$ in roughly the same way that line bundles geometrise integral classes in $H^{2}$. There are several different descriptions of gerbes.

One such description is in terms of line bundles on the loop group of $G$. By transgression (see, e.g., [24]) integral classes in $H^{3}(G)$ are in bijective correspondence with integral classes in $H^{2}(L G)$, where $L G$ is the loop group of $G$. Therefore one can understand a gerbe in $G$ as a line bundle on $L G$. This description is useful in discussing the functional integral approach to the WZW model "16\|. This line bundle can be trivialised locally on the subspace $L Q \subset L G$ corresponding to loops in the conjugacy class; but trivialising a gerbe does not give rise to a line bundle on $Q$.

To see this one needs a different description of a gerbe, in terms of (locally defined) line bundles on $G$ [25]. Suppose that $\mathcal{U}=\left\{U_{\alpha}\right\}$ is an open cover for $G$ such that the restriction of the 3-form $H$ to $U_{\alpha}$ is exact: $\left.H\right|_{U_{\alpha}}=d B_{\alpha}$. The gerbe on $G$ characterised topologically by the integral class $[H] / 2 \pi$ in $H^{3}(G)$ can be defined by specifying a line 
bundle $L_{\alpha \beta}$ on each double intersection $U_{\alpha} \cap U_{\beta}$, an isomorphism $L_{\alpha \beta} \cong$ $L_{\beta \alpha}^{-1}$, and some further conditions in triple and fourfold intersections which will not concern us here. These line bundles come equipped with a natural connection whose curvature is given by $f_{\alpha \beta}=B_{\alpha}-B_{\beta}$.

Now let $\mathcal{V}=\left\{V_{\alpha}=U_{\alpha} \cap Q\right\}$ be an open cover for $Q$ induced from the one for $G$. On $V_{\alpha}$ define $F_{\alpha}=B_{\alpha}-\omega$, since the 2-form $\omega$ is globally defined on $Q$. Clearly $d F_{\alpha}=0$, and it is this that is interpreted as the curvature of the hypothetical line bundle on $Q$. However the curvature is not globally defined on $Q$ : on $V_{\alpha} \cap V_{\beta}, F_{\alpha}-F_{\beta}=f_{\alpha \beta}$. If the class of $H$ is nontrivial, neither is $f_{\alpha \beta}$ and $F_{\alpha}$ and $F_{\beta}$ are therefore curvatures on topologically different line bundles. This means that the line bundles of which the $\left\{F_{\alpha}\right\}$ are the curvatures do not patch up to a global line bundle.

Let us illustrate this for $G=\mathrm{SU}(2) \cong S^{3}$, where $Q \cong S^{2}$ is a spherical conjugacy class. It separates $G$ into two hemispheres $G_{ \pm}$with common boundary $Q$. Let $U_{ \pm}$be open sets defined as the complement of chosen points in the interior of $G_{\mp}$ respectively. For example, if we think of $Q$ as a "parallel", we can take $U_{+}$to be the complement of the south pole and $U_{-}$to be the complement of the north pole. The intersection is $U_{+} \cap U_{-} \cong Q \times \mathbb{R}$. On $U_{ \pm}$we have $H=d B_{ \pm}$, and on $U_{+} \cap U_{-}$we have $B_{-}-B_{+}=f_{+-}$, which is the curvature of a line bundle defined on $Q \times \mathbb{R}$. The restriction of this line bundle to $Q$ is topologically nontrivial provided that $H$ is as well.

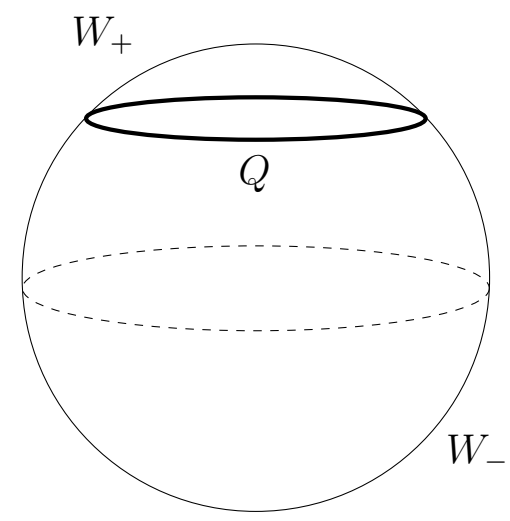

Figure 3. Two ways of computing the D0-charge of a spherical D2-brane $Q$ in $\mathrm{SU}(2)$.

To see this, let $W_{ \pm}$be closed sets contained in $U_{ \pm}$covering $G$ and such that $W_{+} \cap W_{-}=Q$ as shown in Figure 3. We then integrate to find:

$$
\int_{G} H=\int_{W_{+}} H+\int_{W_{-}} H=\int_{Q} B_{+}-\int_{Q} B_{-}=\int_{Q} f_{+-} .
$$

Since $\int_{G} H=2 \pi k$, it follows that the first Chern class of the line bundle whose curvature is $f_{+-}$is $k$ times the generator of $H^{2}(Q) \cong \mathbb{Z}$. This 
provides another way to understand why for the $\mathrm{SU}(2)$ D2-branes, the flux is only defined modulo $k$.

\section{Conclusions}

In this paper we have re-examined the issues of D0-charge and flux quantisation in the context of D-branes in WZW models. We have started by studying the topological conditions necessary for the existence of a consistent theory of open and closed strings propagating on a Lie group. The topological conditions are phrased naturally in terms of the (relative) cohomology of the Lie group modulo the Dsubmanifold on which the D-branes wrap. Table 11 summarises these conditions. The upshot is that that both $H^{2}(G)$ and $H^{2}(G, Q)$ must vanish and that not just must the NS 3 -form $H / 2 \pi$ represent a class in $H^{3}(G ; \mathbb{Z})$, but also the pair $(H, \omega) / 2 \pi$ must represent a class in the relative cohomology $H^{3}(G, Q ; \mathbb{Z})$.

For $G$ be a compact simple Lie group and $k$ the level of the corresponding WZW model, the D0-charge is the class in $H^{3}\left(G, Q ; \mathbb{Z}_{k}\right)$ induced by the reduction modulo $k$ of the relative class represented by $(H, \omega) / 2 \pi$ in $H^{3}(G, Q ; \mathbb{Z})$. We then showed that the class in $H^{3}(G ; \mathbb{Z})$ represented by $H / 2 \pi$ is the obstruction to the existence of a line bundle on $Q$ whose first Chern class induces the relative class $[(H, \omega)] / 2 \pi$. Hence if $H$ is not cohomologically trivial, there is no canonical line bundle on $Q$, and hence no $\mathrm{U}(1)$ gauge field whose flux computes the D0-charge. Instead we have a family of (locally defined) line bundles on $Q$, interpreted as a trivialisation on $Q$ of the gerbe whose characteristic class is $[H] / 2 \pi$, whose Chern classes are equivalent modulo $k$ and such that they agree with the D0-charge. We conclude therefore that for nontrivial $H$, there is no line bundle on the D-submanifold and hence no associated $\mathrm{U}(1)$ gauge theory on all of $Q$. Nevertheless there are locally-defined line bundles and gauge fields whose fluxes are quantised (and defined modulo $k$ ) in such a way that they agree with the D0-charge.

This local picture of the gauge theory suggests a physical situation in which the gauge fields are indeed generated locally where the strings hit the D-brane, but in such a way that they patch up globally only modulo $k$. It would be nice to have independent confirmation of the modularity of the D0-brane charge.

Although we have concentrated in the WZW model, where $G$ is a Lie group, many of the results in this paper are valid in more general situations where $G$ is a riemannian manifold with a nontrivial NS 3-form. The question of the nature of the "gauge field" on the D-submanifold in the presence of a nontrivial NS 3-form has been studied also in [17, 26, 27] and our work gives a complementary perspective on this issue. 


\section{REFERENCES}

[1] C. Bachas, M. Douglas, and C. Schweigert, "Flux stabilization of D-branes," J. High Energy Phys. 05 (2000) 048. arXiv:hep-th/0003037.

[2] J. Pawelczyk, " $S U(2)$ WZW D-branes and their noncommutative geometry from DBI action." arXiv:hep-th/0003057.

[3] W. Taylor, "D2-branes in B fields," J. High Energy Phys. 07 (2000) 039. arXiv: hep-th/0004141.

[4] A. Kling, M. Kreuzer, and J. Zhou, " $S U(2)$ WZW D-branes and quantized worldvolume $U(1)$ flux on $S^{2}$." arXiv:hep-th/0005148.

[5] A. Alekseev, A. Mironov, and A. Morozov, "On B-independence of RR charges." arXiv:hep-th/0005244.

[6] D. Marolf, "Chern-Simons terms and the three notions of charge." arXiv: hep-th/0006117.

[7] S. Stanciu, "A note on D-branes in group manifolds: flux quantisation and D0-charge." arXiv:hep-th/0006145.

[8] A. Alekseev and V. Schomerus, "RR charges of D2-branes in the WZW model." arXiv:hep-th/0007096.

[9] O. Pelc, "On the quantization constraints for a D3 brane in the geometry of NS5 branes." arXiv:hep-th/0007100.

[10] D. Marolf, "Half-branes, singular brane intersections, and Kaluza-Klein reduction." arXiv:hep-th/0007171.

[11] A. Alekseev and V. Schomerus, "D-branes in the WZW model," Phys. Rev. D60 (1999) 061901. arXiv: hep-th/9812193.

[12] S. Stanciu, "D-branes in an $A d S_{3}$ background," J. High Energy Phys. 09 (1999) 028. arXiv:hep-th/9901122.

[13] G. Felder, J. Fröhlich, J. Fuchs, and C. Schweigert, "The geometry of WZW branes," 34 (2000) 162. arXiv: hep-th/9909030.

[14] S. Stanciu, "D-branes in group manifolds," J. High Energy Phys. 01 (2000) 025. arXiv:hep-th/9909163.

[15] J. Figueroa-O'Farrill and S. Stanciu, "D-branes in $\mathrm{AdS}_{3} \times S^{3} \times S^{3} \times S^{1}$," J. High Energy Phys. 04 (2000) 005. arXiv: hep-th/0001199.

[16] K. Gawędzki, "Conformal field theory: a case study." arXiv:hep-th/9904145.

[17] D. Freed and E. Witten, "Anomalies in string theory with D-branes." arXiv:hep-th/9907189.

[18] C. Klimčik and P. Severa, "Open strings and D-branes in WZNW models," Nuc. Phys. B488 (1997) 653-676. arXiv: hep-th/9609112.

[19] E. Witten, "Nonabelian bosonization in two dimensions," Comm. Math. Phys. 92 (1984) 455-472.

[20] M. Karoubi and C. Leruste, Algebraic topology via differential geometry. Cambridge University Press, Cambridge, 1987.

[21] R. Bott and L. Tu, Differential Forms in Algebraic Topology. Springer Verlag, New York, 1982.

[22] J. Figueroa-O'Farrill and S. Stanciu, "More D-branes in the Nappi-Witten background," J. High Energy Phys. 01 (2000) 024. arXiv:hep-th/9909164.

[23] J.-L. Brylinksi, Loop Spaces, Characteristic Classes and Geometric Quantization. Birkhäuser, 1993.

[24] A. Pressley and G. Segal, Loop Groups. Oxford, 1986.

[25] N. Hitchin, "Lectures on special lagrangian submanifolds." arXiv:math.DG/9907034.

[26] A. Kapustin, "D-branes in a topologically nontrivial B-field." arXiv:hep-th/9909089. 
[27] P. Bouwknegt and V. Mathai, "D-branes, $B$-fields and twisted $K$-theory," $J$. High Energy Phys. 03 (2000) 007. arXiv: hep-th/0002023.

Department of Mathematics and Statistics The University of Edinburgh EDINBURGH EH9 3JZ SCOTLAND

E-mail address: jmf@maths.ed.ac.uk

Spinoza Institute UTRECHT UNIVERSITY LEUVENLAAN 4 3508 TD UTRECHT The Netherlands

E-mail address: s.stanciu@phys.uu.nl 